\title{
Using Cellular Agents (CA) to Prove Kurzweil's Law of Accelerating Returns (LOAR) Wrong When Using a Series of Paradigm Shifts Trying to Maintain Exponential Growth
}

\author{
Luis F. Copertari \\ Computer Engineering Program, Autonomous University of Zacatecas (UAZ), Zacatecas, México \\ Email: copertari@yahoo.com
}

How to cite this paper: Copertari, L.F. (2020) Using Cellular Agents (CA) to Prove Kurzweil's Law of Accelerating Returns (LOAR) Wrong When Using a Series of Paradigm Shifts Trying to Maintain Exponential Growth. Open Access Library Journal, 7: e6255.

https://doi.org/10.4236/oalib.1106255

Received: March 23, 2020

Accepted: April 11, 2020

Published: April 14, 2020

Copyright $\odot 2020$ by author(s) and Open Access Library Inc.

This work is licensed under the Creative Commons Attribution International License (CC BY 4.0).

http://creativecommons.org/licenses/by/4.0/

\begin{abstract}
Objective: To prove LOAR wrong with a mathematical and algorithmically designed experiment. Methodology: Using changes from one to two to three to four to five and to six dimensions as paradigm shifts when CA growth occurs. Results: A series of S-curves are obtained and the actual growth of CA is shown. Limitation: The results could not be continued due to the experimental design used. Conclusion: There is evidence against a continued exponential growth even with paradigm shifts being considered.
\end{abstract}

\section{Subject Areas}

Technology, Computer Science, Mathematics

\section{Keywords}

Cellular Agents (CA), Law of Accelerating Returns (LOAR), Theory of Constraints

\section{Introduction}

This paper is about something called exponential growth. Typically, there are three types of growth: arithmetic growth, polynomial growth and exponential growth. Arithmetic growth is simply linear growth. Equation (1) shows the general form for arithmetic growth.

$$
y=f(x)=a+b x
$$

Equation (1) has the typical form of a straight line, where $a$ is the point in 
which the line cuts the vertical axis $(y)$ and $b$ is the slope.

Polynomial growth refers to all kinds of polynomial functions. Polynomial functions have the form shown in Equation (2).

$$
y=f(x)=a_{0} x^{0}+a_{1} x^{1}+a_{2} x^{2}+a_{3} x^{3}+\cdots+a_{n} x^{n}
$$

Equation (2) is a polynomial of degree $n$, that is, the greatest power to which the independent variable $(x)$ is raised to is $n$.

Finally, there is exponential growth. The general form for exponential growth is given in Equation (3).

$$
y=f(x)=a(b)^{x}
$$

In Equation (3), the independent variable $(x \geq 0)$ is the one in the exponent, where $b$ is a given constant and $a$ is the value the dependent variable $(y)$ takes when $x=0$. If $b>1$, then we have exponential growth, whereas if $0<b<1$ we have exponential decay.

Figure 1 illustrates all forms of growth: linear, cubic (polynomial) and exponential. Notice in Figure 1 that linear growth is the first one to appear to grow faster, whereas cubic growth is the second one and exponential growth seems to be the slowest. However, cubic growth soon surpasses linear growth and eventually, exponential growth surpasses even cubic growth. After $x$ is large enough, exponential growth is the fastest to grow, followed by cubic growth and finally by linear growth.

As a matter of fact, exponential growth grows so fast that the best way to depict it is by using what is called a logarithmic scale on the vertical axis $(y)$. In a logarithmic scale base 10, the equal distances in the vertical axis proceed as follows: $10^{0}=1,10^{1}=10,10^{2}=100,10^{3}=1000,10^{4}=10,000$, and so on. Figure 2 shows the same exponential growth displayed in Figure 1 but using a logarithmic scale base 10. Notice that in Figure 2, exponential growth is shown as a straight line on a logarithmic scale. A linear growth or even a cubic growth

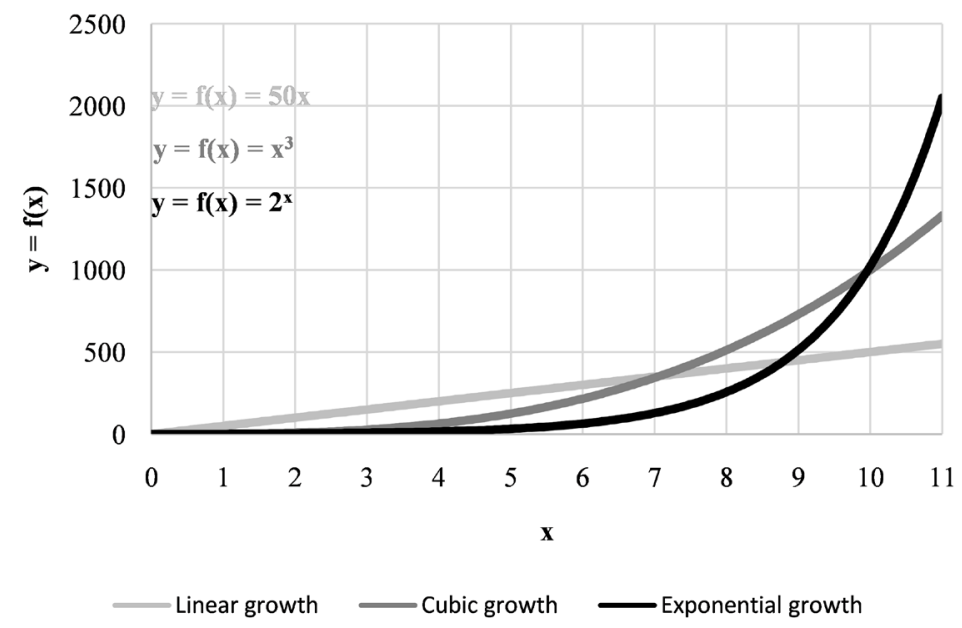

Figure 1. All three main forms of growth: linear, cubic (polynomial) and exponential. 
would not even barely show above the horizontal axis $(x)$.

Growth greater than exponential would show as a curve with increments larger than a straight line, whereas growth lower than exponential growth would show with decrements lower than a straight line, as shown in Figure 3.

In Figure 3 we see in black exponential growth given as the function $y=f(x)$ $=2^{x}$. We also see in light gray lower than exponential growth in the form $y=f(x)$ $=2^{\sqrt{x}}$. Finally, we see greater than exponential growth in dark gray of the form $y=f(x)=2^{x^{2}}$. Notice that lower than exponential growth looks like a decreasing (concave) curve, whereas greater than exponential growth looks like an increasing (convex) curve. Simple exponential growth looks like a straight line. As a consequence, if in a logarithmic scale base 10 we see a concave curve it means such growth is slower than exponential.

Exponential growth is typical of unicellular bacterial growth in Biology [1] [2], compound interest rate growth in Finance and Economics [3], as well as all kinds of technological and knowledge based-improvements in Computer Science.

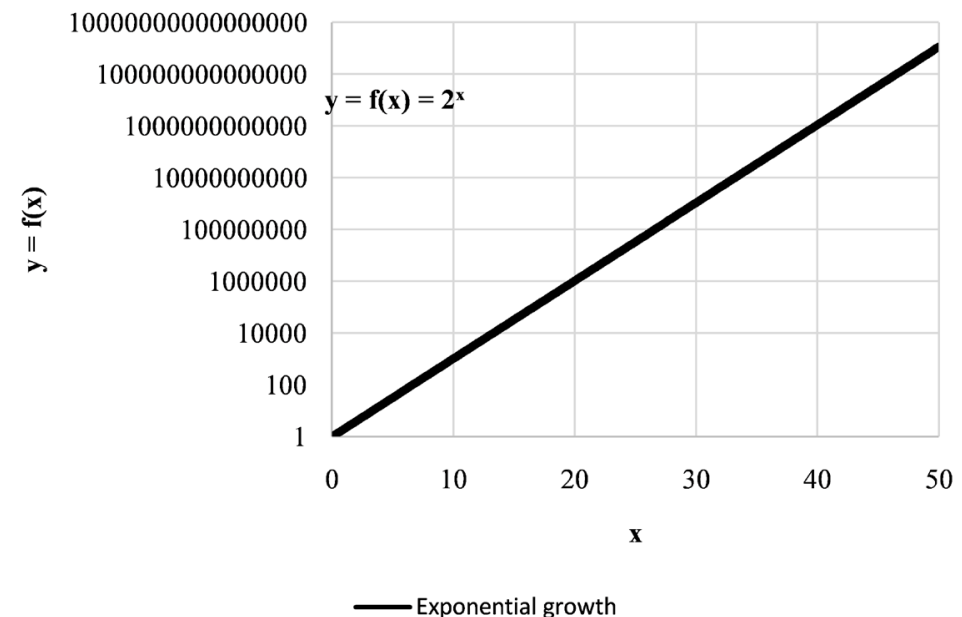

Figure 2. Exponential growth on a logarithmic scale base 10 .

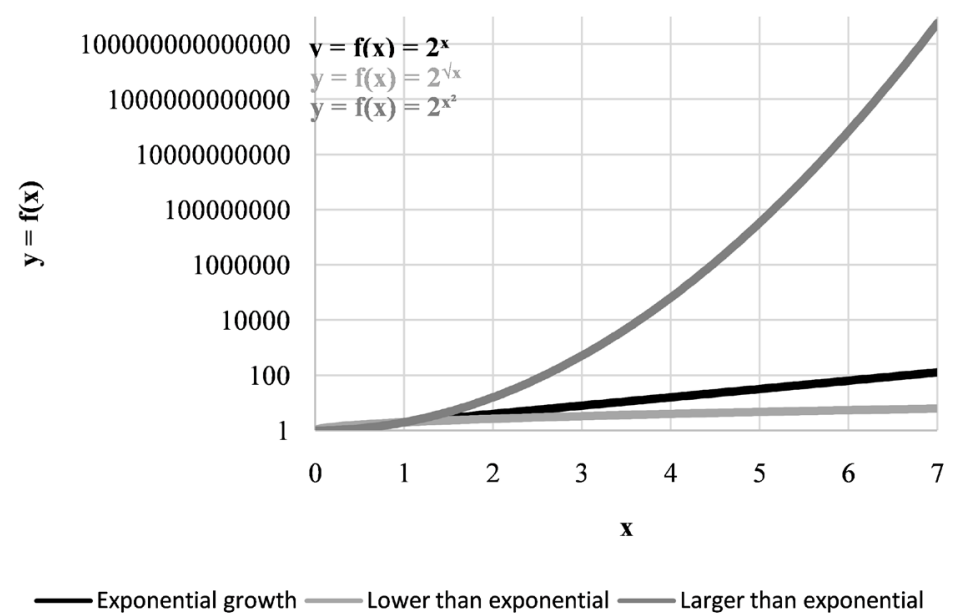

Figure 3. Exponential growth, larger than exponential and lower than exponential growth on a logarithmic scale base 10 . 
However, these types of exponential growth have limits. It is the attempt of this paper to highlight the problem when assuming exponential growth will continue forever.

\section{What Are Cellular Agents (CA)?}

Cellular Agents (CA) are the digital equivalent of bacterial cells. Bacterial cells double every generation as long as their environment is comfortable enough for their procreation. CAs are individual cells occupying one pixel in a discrete (and possible through mathematical and algorithmically generalization multidimensional) space that duplicate in every generation.

In Figure 4 we see the duplication of one single CA, labelled with the number 1, in a two-dimensional space through five generations without the need to slow down its growth. In a two-dimensional space, the CA will try to duplicate by occupying its top pixel, then its right pixel, then its bottom pixel, and finally its left pixel. In the experiments carried out later on we consider occupying top, top-right, right, right-bottom, bottom, bottom-left, left and top-left pixels when considering a two-dimensional space.

In any case, in Figure 4 we see that CA 1 tries to double its number and does so by occupying the top pixel and labelling such pixel with the number 2, indicating such CA belongs to the second generation. Then, for a third generation (where the resulting pixels are labelled with the number 3), both pixel 1 and pixel 2 duplicate. Pixel 1 needs to occupy the right pixel because the top one is already occupied by pixel 2. Pixel 2 occupies the top pixel. At the beginning we had only one pixel $\left(2^{0}=1\right)$, then we had two pixels $\left(2^{1}=2\right)$. Now we have four pixels $\left(2^{2}=4\right)$. After that, all pixels try to duplicate into a fourth generation (labelled with the number 4). All pixels duplicate and occupy their corresponding places (we have now $2^{3}=8$ pixels). Finally, it is possible to have a fifth generation, which is shown with the label 5 (having now a total of $2^{4}=16$ pixels). Clearly, it is not possible to sustain this kind of exponential growth with these rules any longer because pixel 3 at the right side of pixel 1 , for example, has no

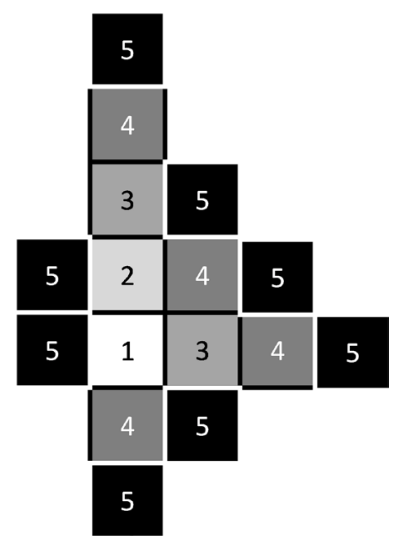

Figure 4. Cellular agent growth in two dimensions spanning five generations. 
place to duplicate. If we were to slightly change the rules by also allowing to occupy the diagonal pixel spaces (having instead of 4 possible spaces to occupy, a total of 8 possible spaces to occupy in a two-dimensional arrangement, we may continue, perhaps, with a sixth generation, but certainly not much further than that.

\section{Kurzweil's Law of Accelerating Returns (LOAR)}

Ray Kurzweil [4] proposed in his book "The age of spiritual machines: When computers exceed human intelligence" that there is a rate of exponential growth in many aspects of technology, going from the number of transistors fitting in an electronic chip (see Figure 5 for what is known as Moore's Law), to the number of phone calls in the United States going from 1890 to 2000, to the number of estimated cell phones subscribers in the United States going from 1985 to 2003, processor performance measured in Million Instructions per Second (MIPS) going from 1971 to 2000, microprocessor clock speed going from 1976 to 2016, the number of total bits stored (shipped) going from 1971 to 2003, magnetic data storage going from 1952 to 2000, among others. These growth rates approximately follow an exponential growth rate, that is, when plotted on a logarithmic (base 10) vertical axis they tend to follow a straight line. However, some of these charts do not exactly follow a straight line on a logarithmic scale, so it is unclear whether or not such trend will continue for a very long time.

In his book "The singularity is near: when humans transcend biology", Ray Kurzweil [5], to the criticism that instead of exponential growth, we actually have S-curves when seen on a linear scale, argues that just when the S-curve is actually reaching its limit, there is a paradigm shift that takes the S-curve to a faster pace, so that overall, the general behavior, seen on a linear scale (like the one on Figure 1), looks like an exponential curve.

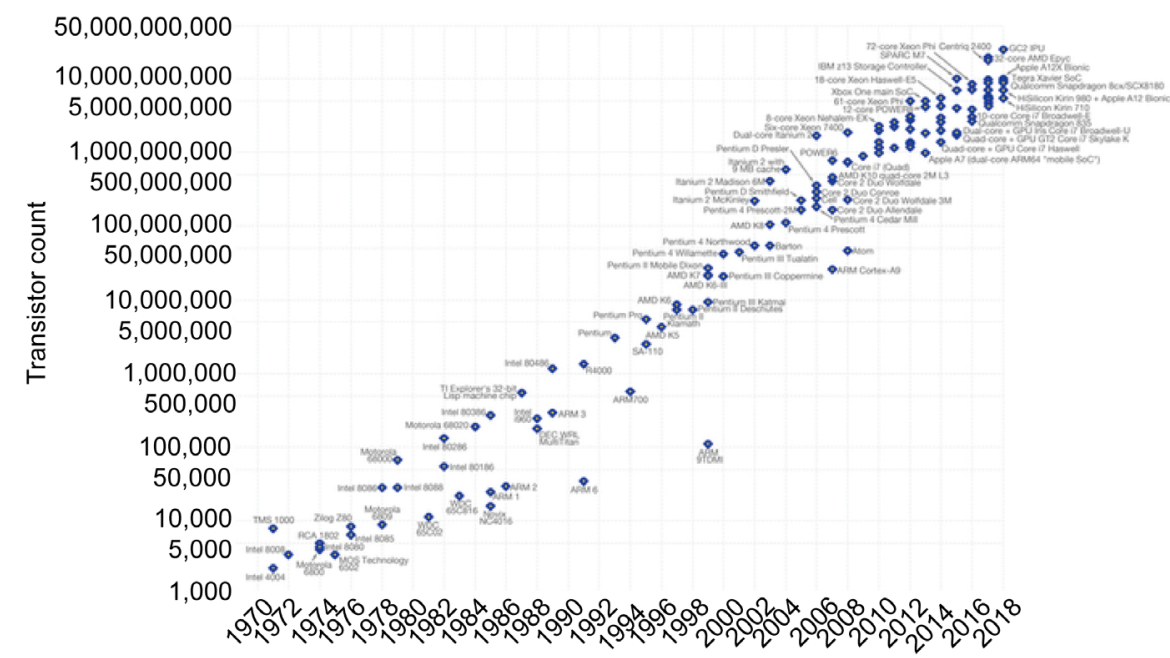

Figure 5. Moore's law in the number of transistors fitting on a chip from 1971 to 2018 plotted on a base 10 logarithmic scale. 
He claims that this is a general trend seen in all kinds of technological and information progress, and not just a coincidence.

\section{Methodology}

In order to prove whether or not a paradigm shift can actually carry on with the exponential growth when a given technology is reaching its limit and gets substituted by a better technology, we are going to use Cellular Agents (CAs) and consider the next paradigm having an additional dimension to use. We start with a grid of one dimension with 16 pixels, continue with the grid of two dimensions with $16 \times 16=256$, then a three-dimensional grid with $256 \times 16=$ 4096 pixels, then a four-dimensional grid with $4096 \times 16=65,536$ pixels, then a five-dimensional grid with $65,536 \times 16=1,048,576$ pixels and finally a six-dimensional grid with $1,048,576 \times 16=16,777,216$ pixels. The paradigm shifts occur when a new dimension is added to the grid. Still, the CAs are constrained by the border imposed by the grid when they try to duplicate in each iteration for each dimension used.

\section{Results}

The first cell agent starts somewhere in the middle of a one-dimensional grid with 16 pixels. Remember that CAs duplicate in every available space they find. Figure 6 shows the linear behavior of such one-dimensional CA.

Table 1 shows the numerical behavior of this kind of CA duplication every generation that it is possible to grow. As can be seen, in iteration 1 there is one CA (the first one), on iteration 2 there are 2 CAs (it has successfully doubled), on iteration 3 there are 4 CAs (it has successfully double once again, but after that the growth increases by two CAs each generation (while there is room on both sides) and then it merely grows linearly when the left corner has been reached. Although this is not exactly an S-curve, it is close enough to it considering there is only one dimension to work with. If true logarithmic (doubling)

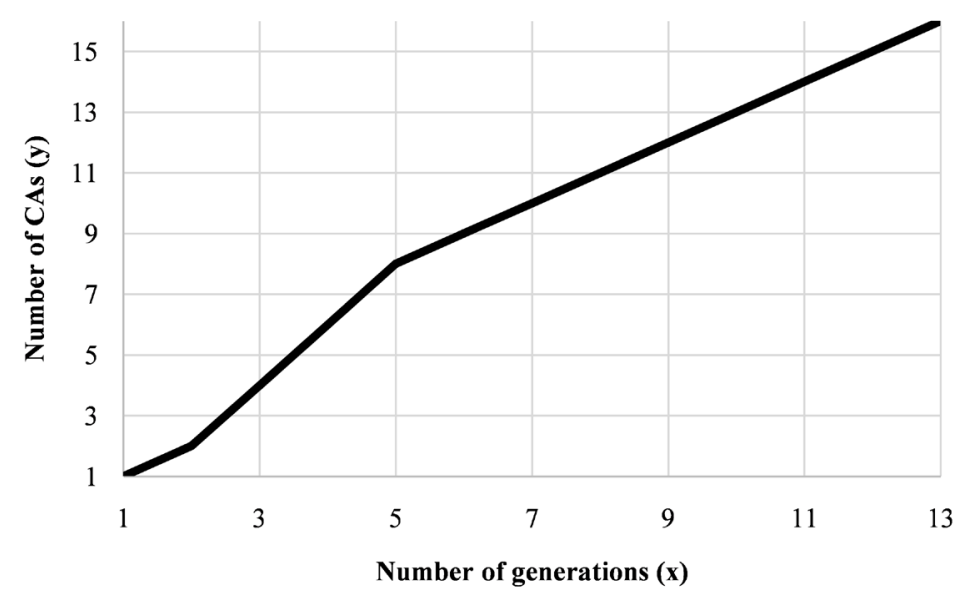

Figure 6. CA trying to double each generation on a one-dimensional grid. 
Table 1. Numerical behavior of the CA growth from Figure 6 on a linear scale.

\begin{tabular}{ll}
\hline $\mathrm{x}$ & $\mathrm{y}$ \\
\hline & 1 \\
2 & 2 \\
3 & 4 \\
4 & 6 \\
5 & 8 \\
6 & 9 \\
7 & 10 \\
8 & 11 \\
9 & 12 \\
10 & 13 \\
11 & 14 \\
12 & 15 \\
13 & 16 \\
\hline
\end{tabular}

growth were to happen, it should have only taken 5 generations (starting with generation zero where there is one CA). Instead, it has taken 13 generations (counting generations from 0 to 12 ).

Then, we make a transition to a two-dimensional grid of 16 by 16 squares. In this case, we begin with all the CAs we had from the previous experiment (16 CAs) and distribute them randomly on the two-dimensional grid. The result of their growth constrained by the borders of the grid is shown in Figure 7.

In Figure 7, we can see the typical S-curve on a linear scale (left axis) and the typical lower than exponential growth of CAs on a logarithmic scale (right axis). Table 2 shows the actual CA growth constrained by the borders of the grid.

In generation 1 we have the original 16 pixels from the one-dimensional grid. In generation 2 the pixels (CAs) have doubled successfully to 32. In generation 3 it has also doubled successfully. However, for generations 4 to 9 it has not. It should have taken 5 generations to double in each generation, but the physical constraints imposed by the borders of the two-dimensional grid do not allow for such behavior.

Then, a new paradigm shift takes place, going from a two-dimensional grid to a three-dimensional grid. This is carried out algorithmically. The result is shown in Figure 8. We can see faster growth than the one from Figure 7 in the form of the typical S-shaped curve.

The actual number of CAs is shown in Table 3. We can see that in the first generation, we take the previous $16 \times 16=256$ CAs. These CAs are randomly distributed in the depth (the new dimension) of the three-dimensional cubic grid. In the first such generation we have the previous $256 \mathrm{CAs}$, in the second generation they successfully double to $256 \times 2=512$. In the third generation 


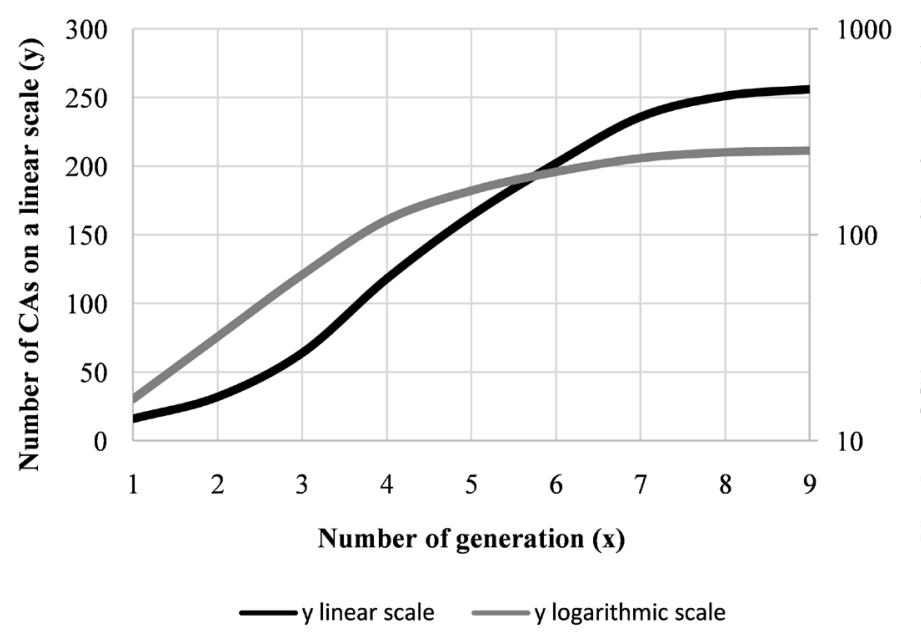

Figure 7. Transition from one dimension to two dimensions with CAs trying to double each generation.

Table 2. Numerical behavior of CA growth from Figure 7.

\begin{tabular}{ll}
\hline $\mathrm{x}$ & $\mathrm{y}$ \\
\hline 1 & 16 \\
2 & 32 \\
3 & 64 \\
4 & 118 \\
5 & 164 \\
6 & 202 \\
7 & 236 \\
8 & 251 \\
9 & 256 \\
\hline
\end{tabular}

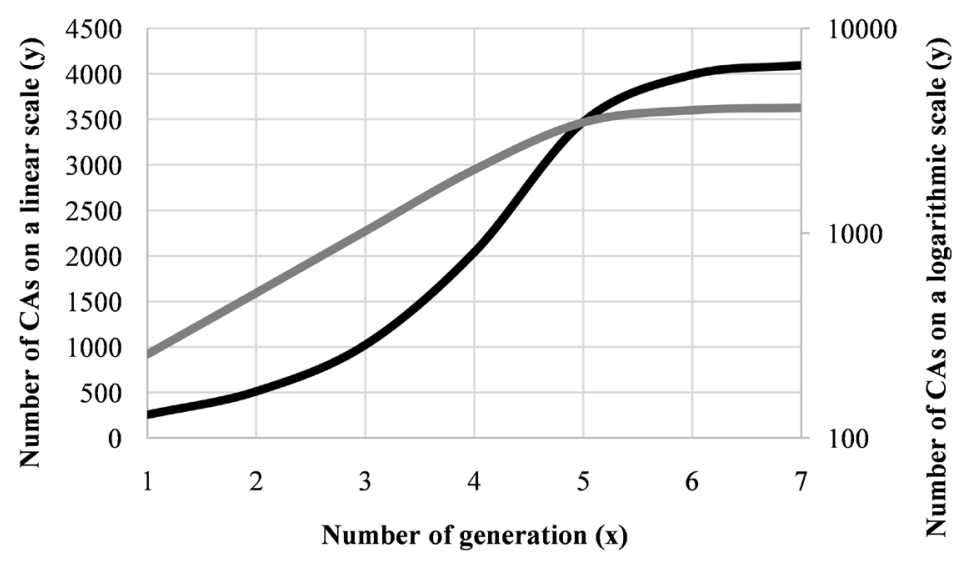

-y linear scale $\quad$ y logarithmic scale

Figure 8. New paradigm shift in the form of a three-dimensional grid. 
Table 3. Numerical behavior of CA growth from Figure 8 (three-dimensional grid).

\begin{tabular}{ll}
\hline $\mathrm{x}$ & $\mathrm{y}$ \\
\hline 1 & 256 \\
2 & 512 \\
3 & 1024 \\
4 & 2044 \\
5 & 3477 \\
6 & 3991 \\
7 & 4096 \\
\hline
\end{tabular}

they double to $512 \times 2=1024$. However, in the fourth generation they fail to double to $1024 \times 2=2048$ and instead reach only 2044, which is still close to the target. After that, the doubling decays.

The CAs multiplicate in a total of 7 generations, where it should have taken them only 5 generation to double if not constrained.

Following, is a shift to a four-dimensional grid (a hypercube). The results are shown in Figure 9. Table 4 shows the actual evolution of the CAs.

The S-curve is clear, and the resulting curve on a logarithmic scale is also characteristic of less than exponential growth. Notice that from Table 3 to Table 4 it takes one additional generation for the CAs to reproduce. That means there has been a slowing of the general trend. Thus, the transition into a new paradigm actually resulted in a worse than exponential performance. The first generation has the previous $4096 \mathrm{CAs}$, then it results in a successful doubling $(4096 \times$ $2=8192)$. The third generation also manages to double $(8192 \times 2=16,384)$. But the fourth generation falls short $(16,384 \times 2=32,768$, where the actual CAs count is 32,759). After that, the CAs loose more and more duplication ability.

Figure 10 and Table 5 show CAs actual evolution for a five-dimensional grid. The information is similar to Figure 11 and Table 6 where the actual CAs evolution is also similar. The four-dimensional, the five-dimensional and the six-dimensional all use the same number of generations for CAs actual behavior (8 generations).

\section{Discussion}

In order to summarize the evolution between transitions of paradigms and within a given paradigm, Figure 12 summarizes the whole process, going from one-dimensional grid to a six-dimensional grid.

Figure 12 neatly summarizes the results. Since the strength of a chain is measured by its weakest link [6] [7], the strongest evidence against LOAR, even with paradigm transitions, is the slow down when going from three-dimensions (a total of 7 steps) to four dimensions, five dimensions and six dimensions (a total of 8 steps each). The apparent acceleration when going to one dimension to two dimensions can be discarded, for being the one-dimensional case too con- 
strained. The black curves show lower than exponential growth, but the gray line shows the minimum growth achieved given the number of steps it takes. According to Goldratt's theory of constraints [6] [7], we should measure the exponential growth based on the weakest links, which occur at the beginning and

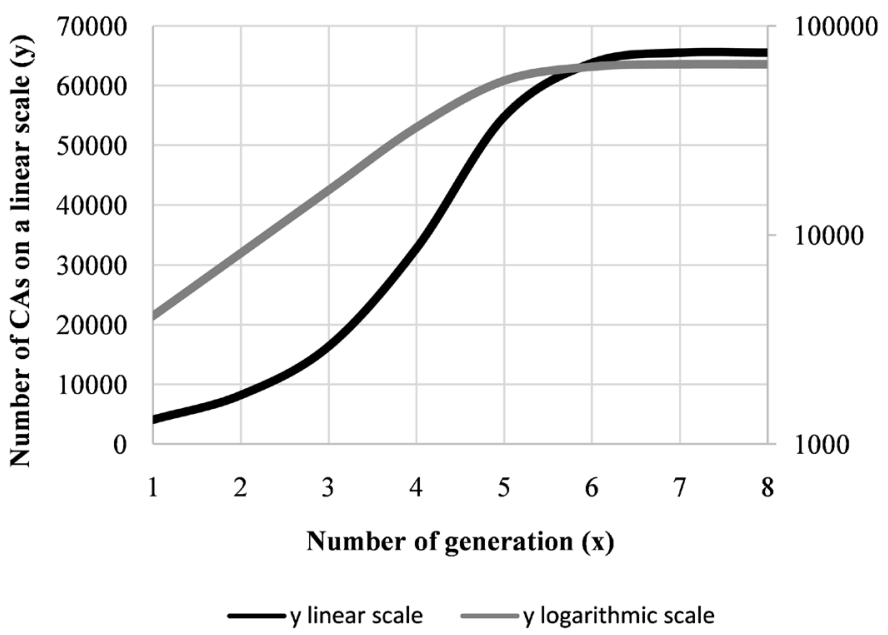

Figure 9. CAs growth on a four-dimensional grid.

Table 4. Actual CAs evolution from Figure 9 (four-dimensional grid).

\begin{tabular}{cc}
\hline $\mathrm{x}$ & $\mathrm{y}$ \\
\hline 1 & 4096 \\
2 & 8192 \\
3 & 16384 \\
4 & 32759 \\
5 & 54797 \\
6 & 63813 \\
7 & 65517 \\
8 & 65536 \\
\hline
\end{tabular}

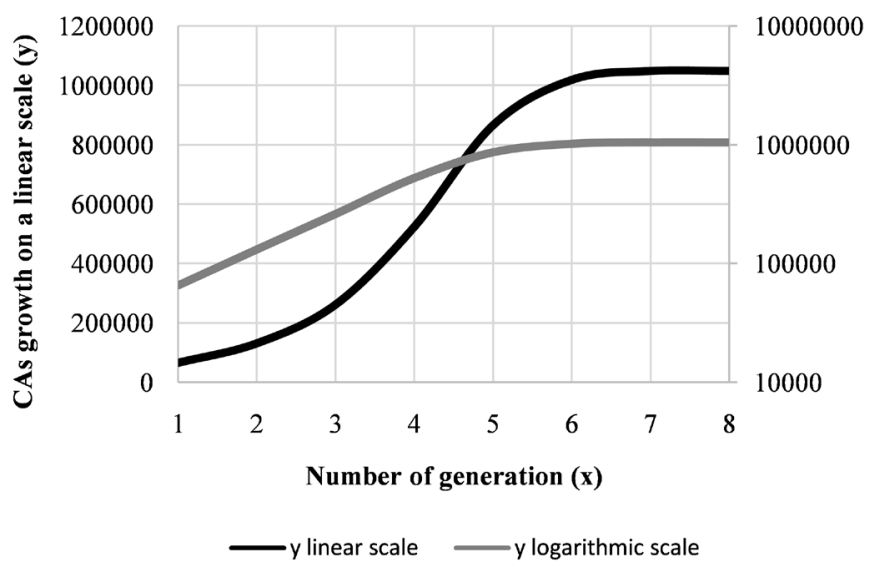

Figure 10. CAs growth on a five-dimensional grid. 
Table 5. CAs actual evolution shown in Figure 10 (five-dimensional grid).

\begin{tabular}{lc}
\hline $\mathrm{x}$ & $\mathrm{y}$ \\
\hline 1 & 65536 \\
2 & 131072 \\
3 & 262144 \\
4 & 524145 \\
5 & 865393 \\
6 & 1019134 \\
7 & 1048478 \\
8 & 1048576 \\
\hline
\end{tabular}

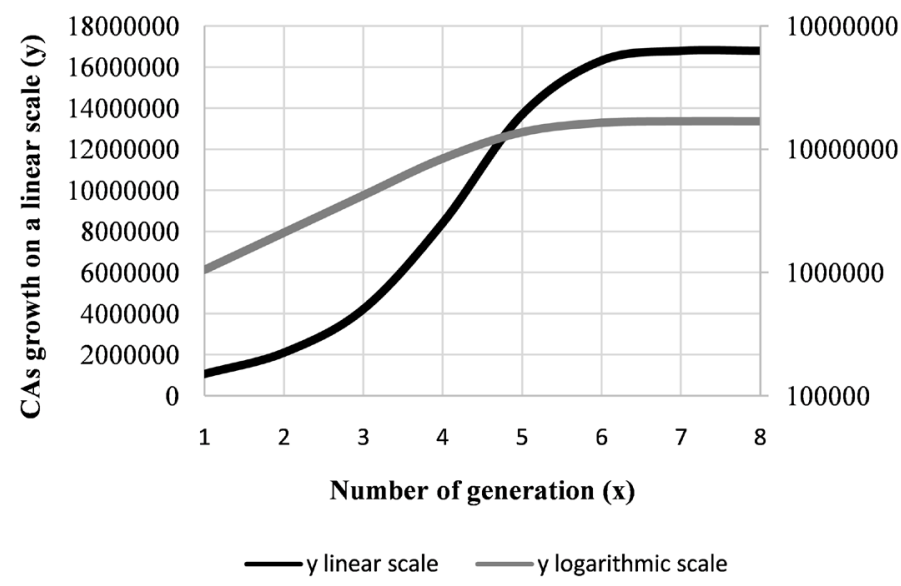

Figure 11. CAs growth on a six-dimensional grid.

Table 6. Actual evolution of CAs growth is shown in Figure 11 (six-dimensional grid).

\begin{tabular}{cc}
\hline $\mathrm{x}$ & $\mathrm{y}$ \\
\hline 1 & $1,048,576$ \\
2 & $2,097,152$ \\
3 & $4,194,304$ \\
4 & $8,386,380$ \\
5 & $1,366,8576$ \\
6 & $16,298,667$ \\
7 & $16,775,994$ \\
8 & $16,777,216$ \\
\hline
\end{tabular}

end of the lower than exponential growth within each paradigm.

There are two accelerations marked by dark gray dots: going from one dimension (13 generations) to two dimensions ( 9 generations) and going from two dimensions ( 9 generations) to three dimensions ( 7 generations). The black dot indicates where a deceleration in growth occurs when going from three dimensions ( 7 generations) to four dimensions and so on ( 8 generations). 


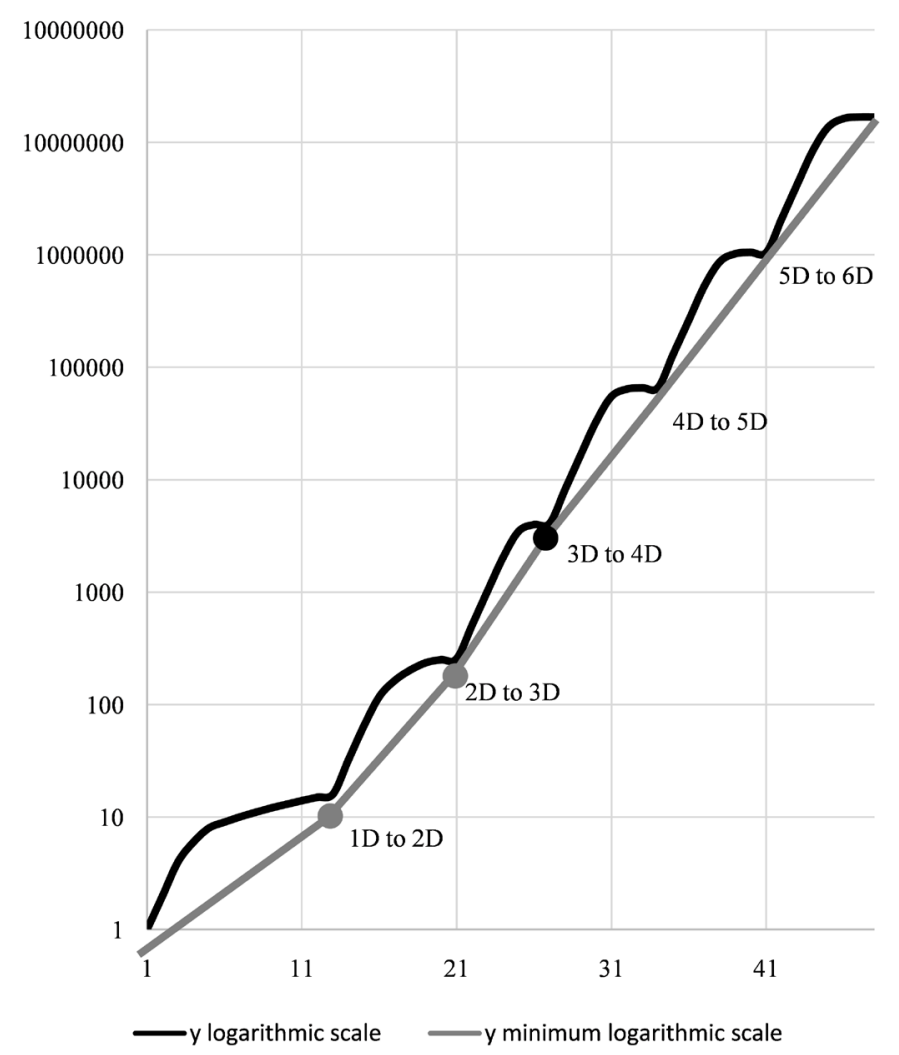

Figure 12. Transitions from paradigm to paradigm and CAs evolution within each paradigm.

\section{Conclusion}

In conclusion, it can be argued that even when taking into consideration paradigm shifts, the evidence presented here tends to favor the idea that continued exponential growth thanks to paradigm shifts is not truly sustainable.

\section{Conflicts of Interest}

The author declares no conflicts of interest regarding the publication of this paper.

\section{References}

[1] Kimball, J.W. (1986) Biología. Addison-Wesley Iberoamericana, Wilmington.

[2] Villee, C.A. (1981) Biología. Nueva Editorial Interamericana, México.

[3] Newnan, D.G. (1983) Análisis Económico en Ingeniería. McGraw-Hill, México.

[4] Kurzweil, R. (1999) The Age of Spiritual Machines: When Computers Exceed Human Intelligence. Penguin Books, New York.

[5] Kurzweil, R. (2005) The Singularity Is Near: When Humans Transcend Biology. PenguinBooks, New York.

[6] Goldratt, E.M. (1990) El síndrome del pajar. Ediciones Castillo, México.

[7] Goldratt, E.M. (1992) La Meta. Ediciones Castillo, México. 